\title{
Determinación del estado ácido-base en mulas de trabajo
}

\author{
Determination of acid-base status in working mules \\ Valeria Trujillo Martínez ${ }^{1}$, Carlos Salazar Latorre, Claudia Valderrama Martínez ${ }^{1,2}$
}

\section{Resumen}

Se realizó un estudio descriptivo para determinar la presentación de alteraciones ácido-base en mulas de trabajo. Se analizaron muestras de sangre de 29 mulares de carga. Se utilizaron tanto la aproximación tradicional (Henderson-Hasselbach) como el modelo de simplificado de diferencia de iones fuertes (Stewart). Los parámetros evaluados fueron $\mathrm{pH}, \mathrm{pCO}_{2}, \mathrm{HCO}_{3}^{-}$, exceso de base, diferencia de iones fuertes, concentración total de aniones buffer no volátiles y lactato sanguíneo. El 48.3\% de los individuos presentó al menos un desorden ácido-base mediante la aproximación tradicional, mientras que el $20.7 \%$ lo hizo mediante la aproximación de Stewart. Las alteraciones más comúnmente presentadas fueron alcalosis metabólica $(20.7 \%)$, alcalosis respiratoria (13.8\%), alcalosis hiperlactatémica (10.3\%) y acidosis de iones fuertes (17.2\%). Los resultados entre las dos aproximaciones no presentaron coincidencia. En conclusión, las alteraciones ácido-base en las mulas de trabajo pueden deberse a factores como el exceso de trabajo, la humedad relativa y la temperatura ambiental del sitio donde se realizó el estudio, que conllevan a marcadas pérdidas electrolíticas, y a la ausencia de suplementación de sales minerales en la dieta de los animales.

Palabras clave: équidos de trabajo; diferencia de iones fuertes; Hendersson-Hasselbach; lactato

\section{AbSTRaCT}

A descriptive study was conducted to describe acid-base alterations in working mules. Blood samples of 29 mules were analyzed and data was interpreted both by the traditional approach (Henderson-Hasselbach) and the Simplified Strong Ion Difference (Stewart). The parameters evaluated were $\mathrm{pH}, \mathrm{pCO}_{2}, \mathrm{HCO}_{3}^{-}$, excess of base, difference of strong ions, total concentration of non-volatile buffer anions and blood lactate. Results

\footnotetext{
${ }^{1}$ Facultad de Ciencias Agropecuarias, Universidad de La Salle, Bogotá, Colombia

${ }^{3}$ E-mail: ccvalderrama@unisalle.edu.co
}

Recibido: 4 de abril de 2018

Aceptado para publicación: 18 de diciembre de 2018 
showed that $48.3 \%$ of the individuals presented at least one acid-base disorder by the traditional approach, while $20.7 \%$ did so by the Stewart's approximation. The alterations most commonly presented were metabolic alkalosis $(20.7 \%)$, respiratory alkalosis $(13.8 \%)$, hyperlactatemia alkalosis (10.3\%) and strong ion acidosis (17.2\%). The results between the two approaches did not show a coincidence. In conclusion, the acid-base alterations in the working mules may be due to factors such as overwork, relative humidity and the environmental temperature, which lead to marked electrolyte losses, and the absence of supplementation of mineral salts in the diet of animals.

Key words: working equids; strong ion difference; Hendersson-Hasselbach; lactate

\section{INTRODUCCIÓN}

Los équidos en países en vía de desarrollo, especialmente las mulas, tienen un papel de gran importancia en la economía campesina, debido a que son una de las fuentes principales de energía de trabajo, siendo utilizadas, especialmente en el sector agrícola, como animales de carga o de tiro (Pritchard, 2010). Colombia es el segundo país productor de panela a nivel mundial, después de India (Osorio, 2007) y la industria panelera en el país ocupa el segundo renglón de la economía agrícola, siendo, después del café, el que más familias emplea, así como el segundo que más mulares utiliza dentro de la línea productiva (DANE, 2016).

Son pocos los estudios dedicados a esta especie, de allí que los realizados en caballos (Equus caballus) son utilizados como información base para los mulares. Dentro de las investigaciones que se realizan en caballos, el equilibrio ácido-base ha sido un tema muy estudiado (Gómez, 2013; van Galen et al., 2013; Jaramillo et al., 2016), mientras que los autores no encontraron estudios en esta área en mulas de trabajo.

El estado ácido base es controlado por los pulmones, los riñones y el sistema buffer. Este último se encuentra distribuido entre la sangre, el fluido intestinal, las células y el hueso. Cuando se presenta un cambio fisiológico, el primer sistema en responder a los cam- bios del estado ácido base es el sistema buffer, el cual está compuesto por una variedad de ácidos débiles que protegerán el metabolismo celular frente a los efectos negativos de las alteraciones ácido base (Fielding y Magdesian, 2015). Entre los participantes más importantes en el sistema buffer se incluyen el ácido carbónico, proteínas extracelulares (como las globulinas, la hemoglobina y la albúmina), iones inorgánicos como fosfato $\mathrm{y}$ sulfato, y los huesos, que contienen un gran reservorio de bicarbonato y fosfato, el cual puede hacer un efecto buffer activo a una carga ácida aguda (Fielding y Magdesian, 2015).

Se han utilizado dos métodos para describir estas alteraciones. La ecuación de Henderson-Hasselbach es ampliamente usada para el manejo clínico de los desórdenes ácido-base; sin embargo, describe la alteración, pero no su mecanismo, y no considera los efectos que tienen otras variables, como las proteínas, sobre el pH (Constable, 2000). El segundo método es el modelo de iones fuertes de Stewart, que tiene un enfoque cuantitativo y que explica cómo el $\mathrm{pH}$ puede afectarse bajo las alteraciones de las proteínas plasmáticas y las concentraciones de fosfato, así como con los cambios en la concentración de iones fuertes, como el sodio y el cloro (Viu et al., 2010).

El presente estudio tuvo como objetivo determinar posibles alteraciones ácido base en mulas de carga un día después de haber 
terminado su jornada de trabajo de seis días con un promedio de 10 horas de trabajo diarias.

\section{Materiales y Métodos}

\section{Consideraciones Éticas}

El estudio fue aprobado por el Comité de Ética de la Facultad de Ciencias Agropecuarias de la Universidad de La Salle, según Acta 157 del día 21 de abril de 2017.

\section{Protocolo de Estudio}

Se realizó un estudio de carácter exploratorio y descriptivo a 29 individuos mulares (15 hembras, 14 machos), con edad promedio de 11 años, utilizados para carga en la cadena productiva de la industria panelera, y alimentados con pasto y subproductos de caña panelera.

El muestreo se llevó a cabo en cinco municipios del departamento de Cundinamarca, donde participaron productores afiliados a la Federación Nacional de Paneleros de Colombia. Los municipios se encuentran ubicados a una altitud promedio de $1200 \mathrm{msnm}$ y presentan una temperatura media de $22^{\circ} \mathrm{C}$ y una humedad relativa de $85 \%$. Los muestreos se hicieron durante cinco lunes, día del descanso de los animales, entre las 09:00 y las 12:00 horas.

\section{Toma y Análisis de Muestras}

A cada individuo se le tomó $5 \mathrm{ml}$ de sangre por venopunción yugular. De esta muestra, $0.5 \mathrm{ml}$ se procesó de forma inmediata en el equipo analizador de gases Epoc Reader ${ }^{\circledR}$ HR-1002-00-00 (Siemens Healthcare) y el resto fue colocado en un tubo con anticoagulante y refrigerada para su transporte al laboratorio clínico de la Clínica Veterinaria de la Universidad de La Salle en Bogotá, Colombia, para la medición de proteínas plasmáticas totales, necesarias para la aproxi- mación de Stewart, utilizando un refractómetro óptico (Scientific ${ }^{\circledR}$ Portable Refractometer, EEUU).

Los resultados obtenidos fueron analizados, tanto por la aproximación de Henderson y Hasselbach, como por el modelo de iones fuertes de Stewart con ayuda de la página de Stewart Acid-Base Analysis en equinos de Ontario Veterinary College (University of Guelph, 2017).

\section{Parámetros Calculados}

El exceso de bases (BE) se calculó utilizando la ecuación descrita por Constable (2000) $\mathrm{BE}(\mathrm{mEq} / \mathrm{l})=0.93 *\left\{\left[\mathrm{HCO}_{3}^{-}\right]-24.4+\right.$ $14.83 *(\mathrm{pH}-7.40)\}$.

\section{Determinación de los Desórdenes Áci- do-Base}

Para la aproximación de HenderssonHasselbach, los desórdenes ácido-base se definieron de la siguiente manera: acidosis metabólica cuando la $\left[\mathrm{HCO}_{3}\right]$ y el BE se encontraban por debajo del rango de referencia ( $24 \mathrm{mEq} / \mathrm{L}$ y $-6 \mathrm{mEq} / 1$ respectivamente); alcalosis metabólica cuando la $\left[\mathrm{HCO}_{3}\right]$ y el $\mathrm{BE}$ se encontraban por encima del rango de referencia $(30 \mathrm{mEq} / 1 \mathrm{y}+6 \mathrm{mEq} / \mathrm{l})$, respectivamente; acidosis respiratoria cuando la $\mathrm{pCO}_{2}$ tenía un valor superior a $53 \mathrm{mmHg}$, alcalosis respiratoria cuando era menor de $41 \mathrm{mmHg}$; acidosis hiperlactatémica si el valor de lactato sanguíneo era mayor de $2 \mathrm{mmol} / 1$ (Viu et al., 2010).

Para la aproximación fisicoquímica de Stewart se definieron: acidosis de iones fuertes (SID acidosis) cuando el valor de SIDm estaba por debajo del valor de referencia (38 mEq/L); acidosis de aniones buffers no volátiles $\left(\mathrm{A}_{\text {tot }}\right.$ acidosis $)$ si el valor de $\mathrm{A}_{\text {tot }}$ estaba por encima del rango de referencia $(16 \mathrm{mEq} / \mathrm{L})$; alcalosis de iones fuertes (SID alcalosis) si el valor de SIDm estaba por encima del valor de referencia $(44 \mathrm{mEq} / \mathrm{L})$, alcalosis de buffers no volátiles ( $\mathrm{A}_{\text {tot }}$ alcalosis) 
Cuadro 1. Resumen de las alteraciones ácido base según cada aproximación

\begin{tabular}{llc}
\hline Aproximación & Alteración & Mulas (n) \\
\hline Tradicional & Normal & 15 \\
& Acidosis respiratoria & 0 \\
& Alcalosis respiratoria & 4 \\
& Acidosis metabólica & 0 \\
& Alcalosis metabólica & 6 \\
& Acidosis hiperlactatémica & 3 \\
& Alcalosis respiratoria + acidosis hiperlactatémica & 1 \\
& & \\
Fisicoquímica & Normal & 23 \\
& SID alcalosis hipoclorémica & 2 \\
& SID acidosis hiponatrémica & 3 \\
& SID acidosis hiperclorémica & 2 \\
\hline
\end{tabular}

si el valor estaba por debajo del rango de referencia (10 mEq/1) (Gómez, 2013).

\section{Análisis Estadístico}

Para todas las variables se determinó la normalidad de los datos mediante la prueba de Shapiro Wilk y se realizó estadística descriptiva utilizando la herramienta RProyect en la plataforma R-studio (RStudio $\left.{ }^{2}, 2018\right)$.

\section{Resultados}

Mediante la metodología de HendersonHasselbach (H-H) se determinó que 14 mulas presentaron alteraciones ácido-base. Se encontró alcalosis metabólica $(\mathrm{n}=6)$, alcalosis respiratoria $(\mathrm{n}=5)$, y acidosis hiperlactatémica $(n=4)$. Uno de los 14 individuos presentó una alteración mixta en la ecuación de H-H (Cuadro 1).

Analizando los datos por medio de la aproximación de Stewart, siete animales presentaron alteraciones: dos con alcalosis SID $\mathrm{y}$ cinco con acidosis SID.
Al evaluar el promedio de los animales por municipio (Cuadro 2), se pudo observar que el valor promedio para las variables de sodio y cloro se encontraban por debajo del rango de referencia en el municipio de Villeta; el valor de BE estuvo por encima del valor de referencia en el municipio La Peña; los valores de $\mathrm{PCO}_{2}$ y lactato estaban por encima de los valores de referencia en el municipio Útica; el valor de SID se encontró por debajo de los valores de referencia en municipio Nimaima; y por último, el valor de $\mathrm{PCO}_{2}$ estuvo por encima de los valores de referencia en el municipio Vergara.

\section{Discusión}

En ausencia de valores de referencia que permitan determinar el estado ácido base en mulas para el análisis de los parámetros evaluados se utilizaron los de caballos reportados en otros estudios (Viu et al., 2010), con el fin de determinar si los valores encontrados bajo la aproximación de Stewart $(\mathrm{Cl}, \mathrm{Na}$, $\mathrm{K}, \mathrm{PPT}, \mathrm{pCO}_{2}, \mathrm{HCO}_{3}^{-}$y lactato) se encontraban dentro de los rangos normales para equinos. 
Cuadro 2. Resumen de los resultados para las variables en estudio (promedio \pm D.E.)

\begin{tabular}{cccccccc}
\hline Parámetro & $\begin{array}{c}\text { Villeta } \\
(5)\end{array}$ & $\begin{array}{c}\text { La Peña } \\
(8)\end{array}$ & $\begin{array}{c}\text { Útica } \\
(6)\end{array}$ & $\begin{array}{c}\text { Nimaima } \\
(4)\end{array}$ & $\begin{array}{c}\text { Vergara } \\
(6)\end{array}$ & $\begin{array}{c}\text { Total } \\
(29)\end{array}$ & $\begin{array}{c}\text { Valores }^{1} \\
\text { referencia }\end{array}$ \\
\hline $\begin{array}{c}\mathrm{pH} \mathrm{epoc} \\
\mathrm{pH}\end{array}$ & $7.42 \pm 0.03$ & $7.44 \pm 0.03$ & $7.41 \pm 0.02$ & $7.44 \pm 0.05$ & $7.40 \pm 0.01$ & $7.42 \pm 0.03$ & $7.31-7.45$ \\
$\begin{array}{c}\mathrm{Stewart} \\
\mathrm{HCO}_{3}-\end{array}$ & $26.43 \pm 0.03$ & $7.41 \pm 0.02$ & $7.37 \pm 0.01$ & $7.41 \pm 0.07$ & $7.36 \pm 0.02$ & $7.39 \pm 0.04$ & $7.35-7.45$ \\
$(\mathrm{mEq} / \mathrm{l})$ & $30.5 \pm 2.4$ & $28.7 \pm 1.5$ & $25.8 \pm 2.5$ & $28.8 \pm 2.7$ & $28.4 \pm 2.7$ & $24.9-31.7$ \\
$\begin{array}{c}\mathrm{BE} \\
(\mathrm{mEq} / \mathrm{l})\end{array}$ & $1.9 \pm 0.9$ & $6.3 \pm 2.6$ & $4.5 \pm 1.6$ & $1.9 \pm 2.4$ & $4.2 \pm 2.8$ & $4.1 \pm 2.7$ & $-6+6$ \\
$\begin{array}{c}\mathrm{Lactato} \\
(\mathrm{mmol} / 1)\end{array}$ & $0.7 \pm 0.4$ & $0.8 \pm 0.2$ & $2.2 \pm 2.1$ & $1.9 \pm 1.1$ & $0.7 \pm 0.3$ & $1.2 \pm 1.2$ & $0-2$ \\
$\begin{array}{c}\mathrm{SID} \\
\mathrm{mmol} / 1\end{array}$ & $38.2 \pm 0.7$ & $41.1 \pm 1.7$ & $40.7 \pm 1.2$ & $37.0 \pm 3.3$ & $40.7 \pm 2.6$ & $40.0 \pm 2.6$ & $38-44$ \\
$\mathrm{~A}_{\mathrm{tot}}$ & $11.4 \pm 1.0$ & $12.6 \pm 0.8$ & $13.2 \pm 1.0$ & $11.7 \pm 0.3$ & $13.1 \pm 0.7$ & $12.5 \pm 1.0$ & $10-16$ \\
$\begin{array}{c}\mathrm{Na}+ \\
\mathrm{mmol} / 1\end{array}$ & $125.5 \pm 6.1$ & $135.2 \pm 2.9$ & $137.3 \pm 3.3$ & $137.6 \pm 0.5$ & $137 \pm 2.0$ & $135.1 \pm 5.0$ & $132-144$ \\
$\begin{array}{c}\mathrm{K}+ \\
\mathrm{mmol} / 1\end{array}$ & $3.9 \pm 0.2$ & $4.0 \pm 0.3$ & $4.2 \pm 0.6$ & $3.7 \pm 0.2$ & $3.9 \pm 0.6$ & $3.9 \pm 0.4$ & $2.9-4.5$ \\
$\begin{array}{c}\mathrm{Cl}- \\
\mathrm{mmol} / 1\end{array}$ & $90.8 \pm 6.4$ & $97.6 \pm 2.9$ & $98.5 \pm 4.7$ & $101.6 \pm 3.0$ & $99.5 \pm 2.0$ & $97.9 \pm 4.8$ & $96-104$ \\
$\mathrm{PPT} \mathrm{g} / 1$ & $62.0 \pm 4.3$ & $70.5 \pm 3.8$ & $75.0 \pm 6.0$ & $66.8 \pm 4.0$ & $75.0 \pm 4.3$ & $70.6 \pm 6.2$ & $55-75$ \\
\hline
\end{tabular}

${ }^{1}$ Valores de referencia tomados de Stämpfli (Stewart Acid-Base Analysis (equine) de Ontario Veterinary College

${ }^{1}$ Viu et al. (2010)

Los valores de $\mathrm{pH}, \mathrm{PCO}_{2}, \mathrm{HCO}_{3}^{-}, \mathrm{BE}$ reportados por Módolo et al. (2017) en mulas anestesiadas fueron inferiores a los valores obtenidos en el estudio presente, probablemente por los efectos generados por los anestésicos. El lactato en cambio se observó superior al promedio obtenido en este estudio. No obstante, los promedios estuvieron dentro de los valores de referencia para equinos reportados por Viu et al. (2010).

La alcalosis metabólica primaria se caracteriza por el aumento en la concentración de bicarbonato en el plasma sanguíneo, o como una respuesta frente a una acidosis respiratoria (Johnson, 1995). En caballos se reporta que es menos frecuente que la acidosis metabólica (Fielding y Magdesian,
2015). En el presente estudio, no obstante, no se encontró casos de acidosis metabólica ni acidosis respiratoria, mientras que 6 de 29 mulas $(20.7 \%)$ presentaron alcalosis metabólica. Las causas de una alcalosis metabólica pueden ser por pérdida de $\mathrm{Cl}$-, exceso de $\mathrm{Na}+$ o deficiencia de $\mathrm{K}+($ Johnson, 1995).

La alcalosis respiratoria se caracteriza por una hipocapnia, debido a un incremento de la excreción pulmonar de $\mathrm{CO}_{2}$ que ocasiona una disminución en la presión de $\mathrm{CO}_{2}$ en el organismo. El descenso de $\mathrm{PCO}_{2}$ genera un descenso en la concentración de $\mathrm{HCO}_{3}^{-}$, a pesar de que no se puede observar en el sistema buffer (Fielding y Magdesian, 2015). La causa fisiológica de este desorden se ve 
asociado a una hiperventilación, asociado además a una termorregulación, hipoxemia, dolor o excitación (Johnson, 1995). En este estudio, 4 de 29 mulas (13.8\%) presentaron este desorden ácido-base. Este fenómeno puede asociarse a la altura presente en la provincia de Gualivá, que oscila entre los 1000 y 1600 msnm y que, por encontrarse en zona tropical, presenta una humedad relativa y una temperatura ambiental elevada, lo cual obliga a los animales a hiperventilar con el fin de regular su temperatura corporal.

La acidosis hiperlactatémica se encontró en cuatro mulas. La hiperlactatemia es la causa de acidosis más común en equinos, teniendo un exceso de lactato en sangre, ya sea por hipoxia secundaria a hipoxemia, hipoperfusión o anemia (Vernon y LeTourneau, 2010). La acidosis encontrada pudo asociarse al exceso de trabajo en los días previos al muestreo o por el transporte (1-2 horas) a través de un terreno montañoso que tuvieron que sobrellevar algunos animales para llegar a al lugar del muestreo. Stull (1999) indica que el lactato es un indicador de fatiga. En este sentido, Yañez-Pizaña et al. (2012) indican que los equinos pueden doblar los valores normales de lactato en un trayecto de dos horas $(<2 \mathrm{mmol} / \mathrm{l})$. En forma similar, Stull y Rodiek (2000) encontraron un incremento del lactato en equinos entre 3 y 6 horas de transporte.

La aproximación de Stewart consta de tres variables independientes $\left(\mathrm{PCO}_{2}\right.$, SID y $\left.\mathrm{A}_{\text {tot }}\right)$ y tres variables dependientes $(\mathrm{pH}$, concentración de hidrogeniones y $\mathrm{HCO}^{-}$) (Constable, 2000). La variable de iones fuertes, dependiente de los electrolitos presentes, es la que más se ve alterada cuando hay presencia de desórdenes ácido-base (Viu et al., 2010). En el presente estudio, se observó acidosis de iones fuertes en 5 de 29 animales (17.2\%), siendo el desorden ácido-base de mayor presentación. La disminución en SID puede ocurrir por una disminución en las concentraciones de sodio o un aumento en las de cloro (Constable, 2000). En el presente estudio, tres animales la presentaron por disminución en sodio y dos por aumento en el cloro. Gómez et al. (2013) reporta la acidosis hiponatrémica como una alteración frecuente en pacientes con procesos diarreicos. $\mathrm{La}$ acidosis hiperclorémica es menos frecuente y se reporta principalmente en pacientes con enfermedad renal como acidosis tubular renal (Stämpfli, 2001).

Por último, una mula presentó alcalosis de iones fuertes, correspondiente a alcalosis hipoclorémica. Una de las causas más comunes de este tipo de alcalosis es el sudor, en el cual se pierden grandes cantidades de ion cloruro (Hoffman et al., 2002; McKeever, 2008; Viu et al., 2010). Llama la atención en este estudio que prime la acidosis hiponatrémica por encima de la alcalosis hipoclorémica, ya que no se encontró evidencia de alguna alteración que llevara a una pérdida patológica de sodio. Una posible explicación, puede ser la poca o nula suplementación de sales mineralizadas que se aporta a los animales del estudio.

\section{Conclusiones}

- La persistencia de alcalosis respiratoria en las mulas de trabajo pudo deberse a factores como la humedad relativa y la temperatura ambiental, además del traslado de los animales al lugar del muestreo, situaciones que llevan a los animales a termorregular a través de la hiperventilación.

- $\quad$ El $48.3 \%$ de las mulas presentó algún tipo de alteración al ser evaluadas mediante la aproximación tradicional, mientras que el $20.5 \%$ las presentó al ser evaluadas mediante la fisicoquímica.

- La aproximación de Stewart, al tener en cuenta más variables para determinar el estado ácido-base de los individuos, permite establecer el origen de los desórdenes ácido base a partir de alteraciones 
en los electrolitos y las proteínas plasmáticas totales. En este estudio solo se vieron desórdenes asociados a los primeros.

\section{Literatura Citada}

1. Constable PD. 2000. Acid-base assessment: when and how to apply the Henderson-Hasselbalch equation and strong ion difference theory. Vet Clin N Am-Food A 29: 115-128. doi: 10.1016/ j.cvfa.2014.03.001

2. [DANE]. Departamento Administrativo Nacional de Estadística. 2016. Encuesta Nacional agropecuaria ENA2016. [Internet]. Disponible en: http:// www.dane.gov.co/files/investigaciones/ agropecuario/enda/ena/2016/ boletin_ena_2016.pdf

3. Fielding C L, Magdesian K. 2015. Equine fluid therapy. USA: WileyBlackwell. 384 p.

4. Gómez DE, Arroyo LG, Stämpfli HR, Cruz LE, Oliver OJ. 2013. Physicochemical interpretation of acidbase abnormalities in 54 adult horses with acute severe colitis and diarrhea. J Vet Intern Med 27: 548-553. doi: 10.1111/ jvim. 12071

5. Hoffman RM, Hess TM, Williams CA, Kronfeld DS, Griewe-Crandell KM, Waldron JE, Graham-Thiers PM, et al. 2002. Speed associated with plasma $\mathrm{pH}$, oxygen content, total protein and urea in a $80 \mathrm{~km}$ race. Equine Vet J 6: 39-43. doi: 10.1111/j.2042-3306.2002.tb05389.x

6. Jaramillo C, Ramírez L, Arias M, Álvarez I. 2016. Gases sanguíneos, electrolitos y variables metabólicas determinantes del estado ácido-base en caballos criollos colombianos. Rev Med Vet Zootec 6: 20-29. doi: 10.15446/ rfmvz.v63n1.56900

7. Johnson PJ. 1995. Electrolyte and acid-base disturbances in the horse. Vet Clin N Am-Equine 11: 491-514. doi: 10.1016/S0749-0739(17)30312-7
8. McKeever K. 2008. Body fluids and electrolytes: responses to exercise and training. In: Equine exercise physiology. Elsevier. p 328-349.

9. Módolo TJC, Munerato MS, Bueno GM, Pereira GT, Marques JA. 2017. Evaluation of intranasal oxygen supplementation in mules anesthetized with the combination of ketamine, butorphanol, and guaifenesin. Arq Bras Med Vet Zoo 69: 130-138. doi: 10.1590/ 1678-4162-9059

10. Osorio G, 2007. Buenas prácticas agrícolas -BPA- y buenas prácticas de manufactura -BPM- en la producción de caña y panela. Medellín, Colombia: Corpoica. $199 \mathrm{p}$.

11. Pritchard JC. 2010. Animal traction and transport in the $21^{\text {st }}$ century: getting the priorities right. Vet J 186: 271-274. doi: 10.1016/j.tvj1.2010.08.004

12. RStudio Team 2018. RStudio: Integrated Development for R. RStudio. Boston, MA. [Internet]. Disponible en: http://www.rstudio.com/

13. University of Guelph. 2017. Stewart acid-base analysis (equine). [Internet]. Available in: https://ovc.uoguelph.ca/doc/ henry-staempfli-acid-base-analysis/14.html

14. Staempfli H. 2001. How to use the routine serum biochemical profile to understand and interpret acid-base disorders in the horse. In: Proc Annual Convention of the AAEP. [Internet]. Available in: https://pdfs.seman-ticscholar.org / c b 88 / fd 4762 b 83 ed a 4b5a29ac7331453113378616.pdf

15. Stull CL. 1999. Responses of horses to trailer design, duration and floor area during commercial transportation to slaughter. J Anim Sci 77: 2925-2933.

16. Stull CL, Rodiek AV. 2000. Physiological responses of horses to 24 hours of transportation using a commercial van during summer conditions. J Anim Sci 78: 1458-1466. doi: 10.2527/ 2000.7861458x 
17. van Galen G, Cerri S, Porter $S$, Saegerman C, Lefere L, Roscher K, Votion DM. 2012. Traditional and quantitative assessment of acid-base and shock variables in horses with atypical myopathy. J Vet Intern Med 27: 186-193. doi: 10.1111/jvim.12003

18. Vernon C, Letourneau JL. 2010. Lactic acidosis: recognition, kinetics, and associated prognosis. Crit Care Clin 26: 255-283. doi: 10.1016/j.ccc.2009.12.007
19. Viu J, Jose-Cunilleras E, Armengou L, Cesarini C, Tarancón I, Rios J, Monreal L. 2010. Acid-base imbalances during a $120 \mathrm{~km}$ endurance race compared by traditional and simplified strong ion difference methods. Equine Vet J 42: 76-82. doi: 10.1111/ j.2042-3306.2010.00213.x

20. Yáñez-Pizaña A, Roldán-Santiago P, Mora-Medina P, Borderas-Tordesillas F, Flores-Peinado S, Mota-Rojas D. 2012. Effects of transport on the metabolism of horses. Rev Cient 22: 432-436. 